\title{
FLIP TEACHING VS COLLABORATIVE LEARNING TO DEAL WITH HETEROGENEITY IN LARGE GROUPS OF STUDENTS
}

\author{
F. Barrio-Parra, M. Izquierdo-Díaz, D. Bolonio, Y. Sánchez-Palencia, \\ L.J. Fernández-Gutiérrez Del Álamo, L.F. Mazadiego \\ Universidad Politécnica de Madrid (SPAIN)
}

\begin{abstract}
High school students start their first year at the university with very different levels of knowledge and skills. Chemistry and Technical Drawing are two appropriate examples to study this complex situation. Due to the flexible high school academic curricula and the lack of strict requirements to enter university degrees in the case of, for example, engineering degrees, it is possible for a student to access without studying Chemistry or Technical Drawing in the last years of high school. The challenge of teaching heterogeneous and large groups has been addressed using different methodologies. In particular, this work has compared two methodologies that can be named as the "classical" and "innovative" approaches. The first one tries to level the students before the standard lessons, applying a flip teaching technique. The second one face heterogeneity, not as problem, but as an opportunity: collaborative learning using working teams tries to level the students through interactions between them. The results of both methodologies have been measured using different external controls: tests, chronological development of activities, continuous assessment questionnaires or final tests. An important conclusion is that both methodologies have a clear impact in the motivation of the students, however this motivation does not well translate into final improvements of the students' marks in the subjects. Flip teaching videos must be done according to the learning objectives and students need to get used to watching videos to prepare the lessons because, in general, it is something new for them. Collaborative learning produces synergy that contributes to the learning process, but interactions between students and teachers must be encouraged. Finally, the authors suggest a critical reflection of the optionality of some subjects in the last years of high school and the possibility to access some degrees without having essential knowledge of some subjects.
\end{abstract}

Keywords: Cooperative, Technical Drawing, Laboratory, Inverted classroom.

\section{INTRODUCTION}

Nowadays, the pre-university education allows access to undergraduate studies without having studied basic subjects such as Chemistry or Technical Drawing. In addition, students come from educational centres with different evaluation and learning methodologies (i.e., "classical" master class vs project based learning). This fact contributes to very heterogeneous groups of first year students, with different experience and skills, which hinders the process of teaching and learning. As a consequence, students are discouraged, frustrated and, in some cases, they drop out of higher education [1]. This fact is enhanced with the high number of students which makes it increasingly difficult to apply a more personalized learning environment, being necessary the application of "virtual environments" to allow collaborative and adaptive learning processes. The "classical" approach to deal with such heterogeneity has the goal of getting all the students to the same level, using the so-called "zero courses", given prior to the start of the lessons [2-6]. Other approaches, like collaborative learning, seek to take advantage of the heterogeneity to improve the learning process (Figure 1). As far as our knowledge, there is a lack of studies that compare both alternative approaches. Thus, the aim of this study is the comparison of the results of two educational innovation projects, which applied each methodology, to large groups of first year engineering students to deal with previous knowledge and experience heterogeneity. 


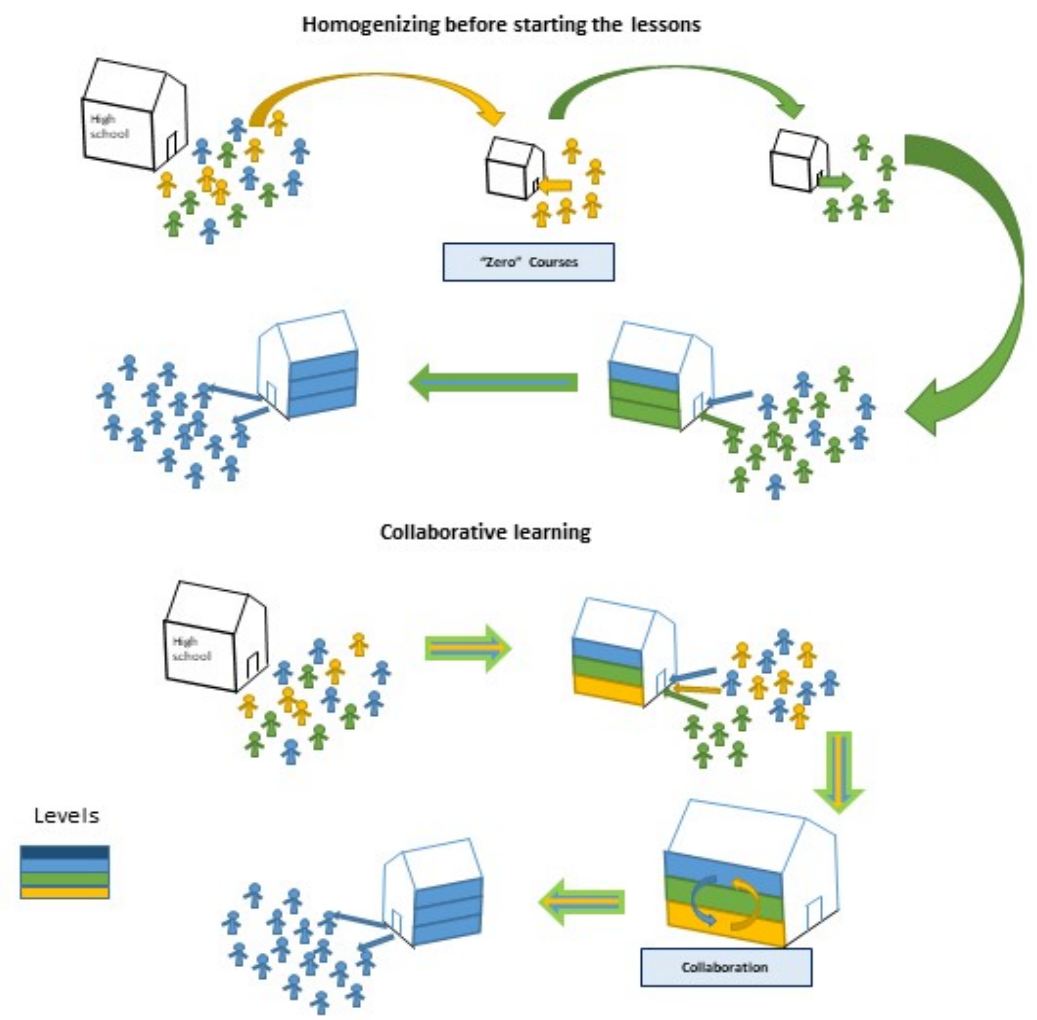

Figure 1. Comparison of collaborative and "classical" methodologies to deal with heterogeneity.

\section{METHODOLOGY}

\subsection{Flip teaching}

First, the aforementioned "classical" approach was adapted to an inverted classroom methodology (or flip teaching), to increase motivation and improve practical skills in the chemistry laboratory [7]. The flip classroom experience was carried out with students enrolled in two different Engineering Degrees. Both groups showed a decrement trend in the final marks during the last years (see section 3.1). As the learning methods and the teachers have not suffered substantial changes during the observational period, a change in the chemistry level of the students is suspected. Until the start of the inverted classroom experience, the students had to make a previous preparation of the practical lessons through the study of some guidelines, where the theoretical fundamentals and the details of the practical execution were described. Right at the beginning of the practical sessions, the laboratory master had to briefly explain the practical issues and doubts raised from the reading of the texts. This fact was a consequence of the lack of graphical (or visual) explanations of the laboratory procedures, that generates anxiety to the students due to the absence of a clear idea of what they have to face in the session. This explanation reduces the available time of the student to face by themselves the chemistry experiments, focusing on the solution of barely practical issues and distracting them from the fundaments of the experiences. Thus, the students were focused on "how to perform the experiment" and were far from the "why the steps were made". The insufficiency of time and high ratio students/teachers avoid a personalized feedback. This status can be partially upgraded by the previsualization of demonstrative videos by the students. Thus, the main objective of the Flip-teaching experience was to improve the understanding of practical procedures and their theoretical foundations by visualizing the development of the laboratory work before the experimental session.

The hypothesis under this method is that the student will reduce their anxiety caused by their lack of practical laboratory experience, allowing them to concentrate on the study of theoretical foundations of the experiments. The videos were uploaded to a YouTube Playlist ("QUIMETUBE: actualizando las prácticas de Química mediante Aula Invertida y TICs") in an institutional channel ("ETSI MINAS Y ENERGÍA UPM Madrid") in order to assess the visualization patterns of the students. To test their understanding, a theoretical-practical online questionnaire was mandatory after watching the videos. Those questionnaires were implemented in a Moodle platform. At the end of the practical session, 
students take an exam with problems related with the theory involved in the experiments performed (Figure 2). If the hypothesis is true, the visualization of the videos and the answer of the previous questionnaires will produce a levelling of the students before the beginning of the practical sessions that would have to rebound in a better average grade of the whole group. To check the validation of the hypothesis a comparison of the student marks was performed with the results of previous courses. The motivation of the students was assessed by a survey designed by the teachers. The experience was performed with first year students from two different engineering degrees. In order to avoid bias due to the differences in the entering scores, the data were analysed separately.

\subsection{Collaborative Learning}

As an "alternative" approach, a collaborative work system was proposed in groups formed by students of heterogeneous level. The aim is to use collective intelligence to take advantage of the heterogeneity of students. The hypothesis is that collaborative work can encourage motivation of the most advantaged students to help their colleagues and pushing low level students to catch up with the average level of the group. The experience, described in Fernández-Gutiérrez del Alamo et al. (2018) [1], employs the physical and temporal student interaction during the lessons and applies ICTs to control the progress of the students (Figure 2).
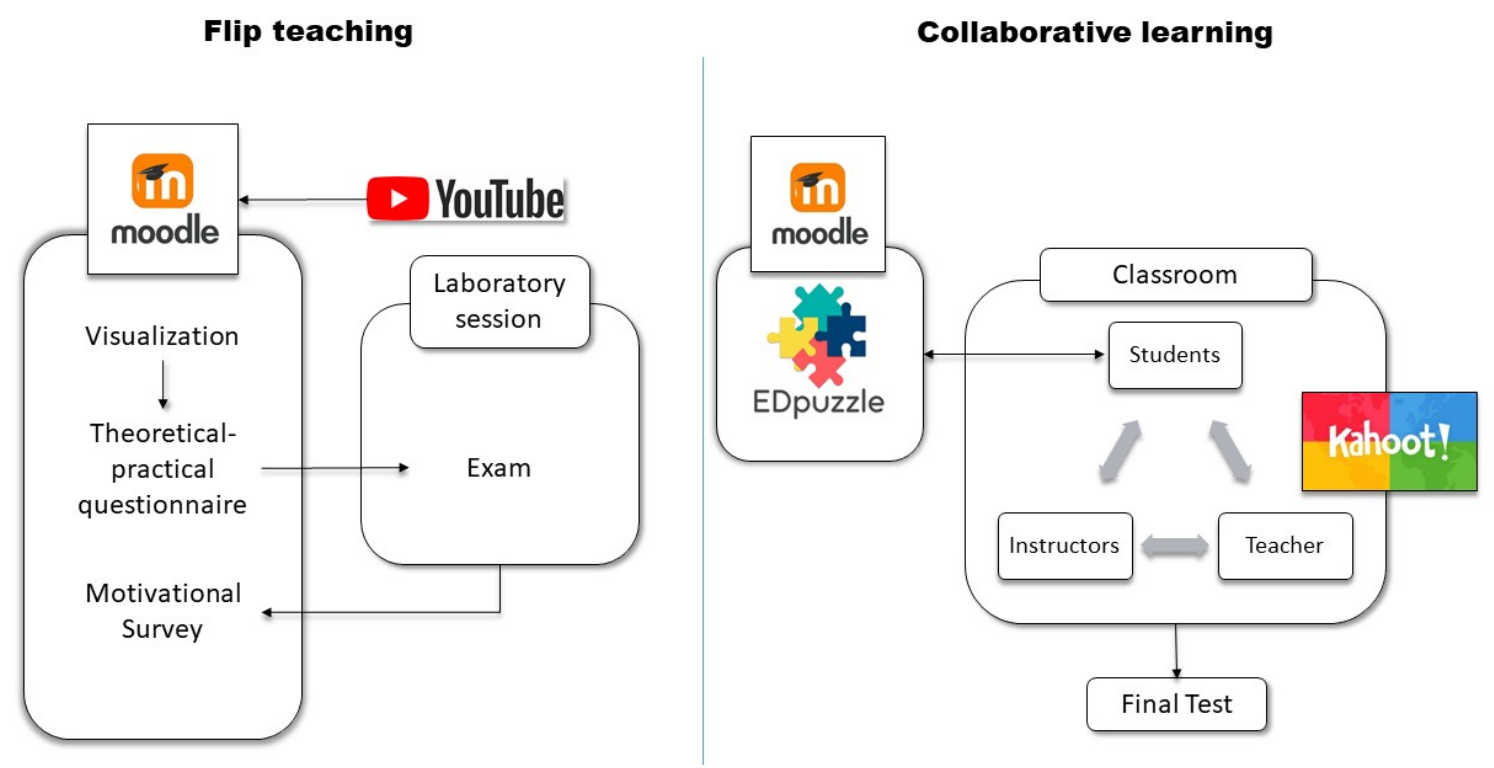

Figure 2. Graphical description of the employed methodologies.

Previous studies $[1-6,8]$ have shown that self-assessment about competence and experience offers reliable data on the level of previous knowledge of the students. Thus, as a previous step, the own perception of the student experience and competence was assessed through a Moodle test developed by the authors, giving information about the initial level of the students in the subject "Technical Drawing". The results of the self-assessed previous knowledge were used to assess the heterogeneity degree of the teams.

Students (of 4 to 6 members) where freely grouped in order to obtain heterogeneous working teams. Groups formed by more than 6 students were considered excessive because some students could easily "hide" in the group. Practical lessons were carried out in classrooms that allow the teams to sit together, pushing the members to collaborate during the resolution of the proposed exercises. The raised doubts were solved both by the teacher and instructors, which were students who already passed the subject during the previous course. The objective of the employment of instructors is to enhance the interactions between students, creating a "relaxed" environment that eases communication of the questions and solutions raised during the execution of the exercises.

The control of the learning progress of the students was performed during the lessons (using Kahoot! tests and exercises during the class, both prepared to give a fast feedback to the students) and outside the classroom (applying Moodle questionnaires and EdPuzzle videos). As a last step, the students' knowledge and skills were assessed by individual and group problems and by an evaluation exam. To handle students' progress data obtained from the Kahoot! tests, EdPuzzle videos, delivered exercises 
and final tests, the ECAM software (which is the acronym for the Spanish name "Evaluación Continua de Alumnado Masivo", that could be translated as "Continuous evaluation of large group of students") was employed.

The average final marks of the students were used to assess the differences between the teams classified by its heterogeneity.

\section{RESULTS}

\subsection{Flip teaching}

A comparison of the final marks obtained in the two exams performed by the two engineering degrees during the last five courses can be seen in Figure 3. As opposed to the expected result, the negative trend observed during the last years has been maintained. The observed visualization pattern is characterized by high peaks that temporally matches the previous days of the laboratory lessons, suggesting that the time dedicated to clarify their doubts and understand the theoretical contents was very limited. Moreover, this result suggests that the students do not re-visualize the videos after the practical lessons or either before their final exams. Thus, it seems that the students watch the videos just to answer the questionnaires previously to begin the laboratory. However, the final survey results suggested that the students considered that this methodology was beneficial, they were more motivated and recommended to continue the use of videos for understanding the theoretical and practical concepts before attending the practical lessons. A detailed description of the obtained results can be consulted in the chapter entitled "The experience of flipped classroom in chemical laboratory classes for engineering students: QUIMETUBE" by Sanchez-Palencia et al. in this volume.

The flip-teaching experience yields several final remarks:

- Practical videos do not improve final marks: The results showed that, despite the students recommendation for the use of videos, it seems that it does not improve the final marks. Figure 4 shows a lack of relationship between the scores obtained in the exams at the end of the laboratory lessons and the number of visualizations. This could be related with the fact that the content of the videos does not teach the students how to solve the problems that they face in the exams. So, if we want to improve the final marks of our students, we should develop videos with contents that help the student to clarify doubts related with the exams or motivate the students to employ the didactic materials more efficiently outside the classroom, which leads to the following point.

- The way students employ the didactic materials is important: the study of the visualization temporal trend have shown us that our students have watched the videos just before the practical lessons and maybe motivated by the obligation of taking a Moodle questionnaire. They do not revisualized the contents prior to taking their exams, maybe because their contents do not match with the solution of theoretical problems (previous point). Thus, it seems important to motivate the students to self-learn outside the classrooms, especially during the pre-university studies, to avoid the academic fail, teaching them to combine the didactical materials that they have to completely fulfil with the didactic competences of each subject.

- The audio-visual materials improve the motivation of the students: as shown by the student survey, the YouTube videos improve their motivation and they recommend the application of this methodology. The mismatch between the final marks and their motivation suggests that, despite they like the audio-visual materials, these do not motivate the students to study. Thus, it seems that this kind of didactic materials approximate the university to the 21st century and the relationships of the new generations with the multimedia content. 
Evaluation \# 1

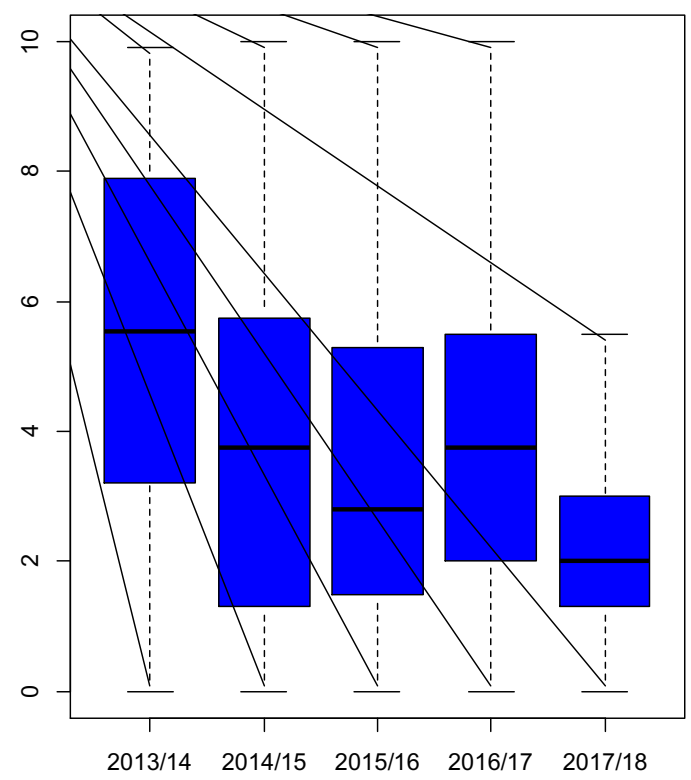

Evaluation \# 2

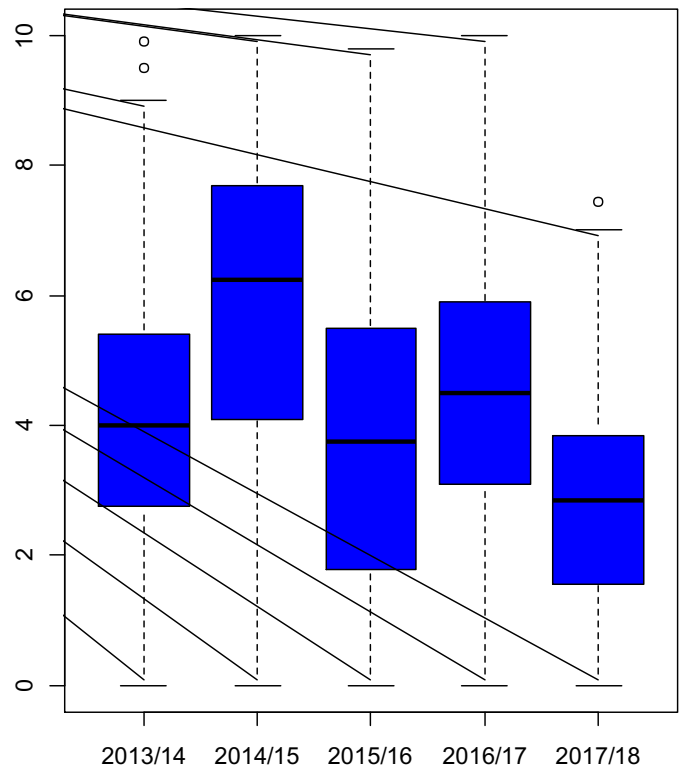

Figure 3. Evolution of evaluation marks in chemistry exams for two Engineering Degrees.

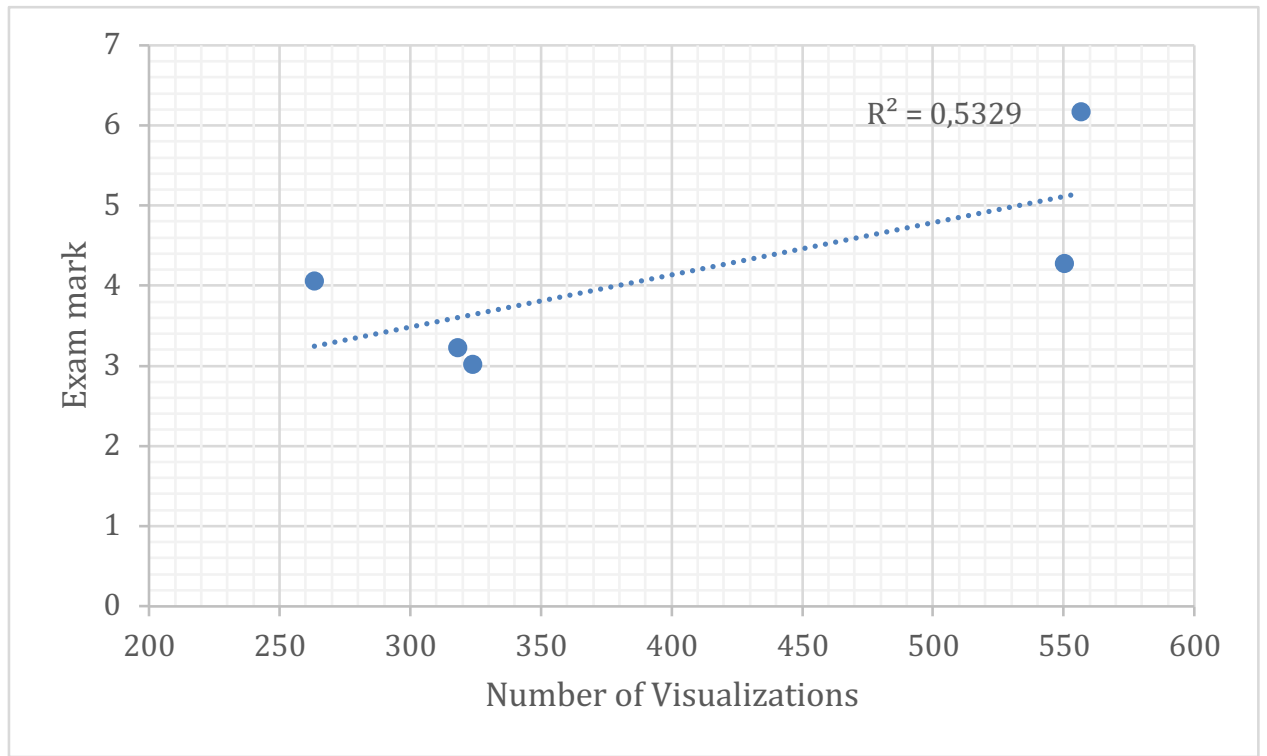

Figure 4. Relationship between the average exam scores and the number of visualizations.

\subsection{Collaborative Learning}

As expected from previous studies [7], the freely arrangement of students formed heterogeneous teams. The heterogeneity of the groups was assessed in base to the results of the self-assessed previous experience and competence and considering the entry score. In the experience, based on collaborative learning, students with low initial level obtained better grades when they were in groups with advantaged students. The students with Low or No previous experience in "Technical Drawing" got average final marks of $8 / 10$ and $10 / 10$ respectively in the teams with high heterogeneity (formed by students with Low, Medium and No experience). Similarly, the mean marks were higher in high heterogeneity teams (8.1/10 and 7.3 in teams formed by Low, Medium and Non Experience members and Low, Medium and High experience respectively) versus groups formed by homogenous initial experience students (i.e., homogeneous groups formed by High experienced students get an average mark of 2.0/10). In addition, students with high initial level got better grades when they were in heterogeneous groups. A detail of the obtained results can be found in Fernández-Gutiérrez del Alamo et al. (2018) [1].

Several remarks can be highlighted from the application of the collaborative learning experience: 
- Students with higher initial experience/competence have higher final marks. As expected, the initial level of the students continues being a determinant factor in the academic success.

- Synergic interactions appear in heterogeneous groups. It seems that the initial hypothesis about the increase of the motivation of high level students when they collaborate with partners with lower previous knowledge or skills is fulfilled. The inverse process is apparent while observing how the low level student increase their final marks when they collaborate with higher level students.

- The environment is important. The communication in the classroom between teachers and students have been improved through the collaboration of instructors. It seems that students feel more comfortable while asking their doubts to other students with higher experience, improving the communication and the learning process.

\section{CONCLUSIONS}

The performance of both flip-teaching and collaborative learning experiences and their comparison highlights several important notes about heterogeneity in large groups of students:

- The collaboration between heterogeneous students produces a synergic positive effect in the learning process while an active strategy is considered (i.e. project based learning, learning based on challenges or problem lessons). To achieve these benefits, the classroom environment should promote the interaction between students and the teachers. To ease this communication, the role of instructors seems to be important.

- The initial level of the students is still a determinant factor in the final marks. The negative trend observed in the chemistry marks and the results of the collaborative learning experience suggest the importance of the initial level of the students. Thus, despite the effectiveness of the applied learning strategies to deal with heterogeneity, the academic success of the students is still dependent on the knowledge and skills acquired during the pre-university studies. Thus, the educational policy should consider the consequences of the optionality of the subjects in the academic development of the students during the pre-degree studies.

- The content of the flip-classroom material should be carefully chosen depending on the learning objectives. If the goal of this strategy is to improve the marks, the content of the videos should be oriented to clarify the student doubts about the resolution of the questions that are going to face in the tests. As suggested by the student survey, the practical orientation of the videos have increased their motivation, which is a really important goal. However, as we have seen in our study, this goal is not directly related with the achievement of higher marks.

- Further work should be done in order to entrench the self-study habits in the undergraduates. The study of the visualization patterns has shown a lack of continuity in the study of the subject contents. Thus, a further effort should be done in order to improve the continuous learning system.

\section{ACKNOWLEDGEMENTS}

We would like to thank the funding obtained through the projects "Sistema de Inteligencia Colectiva Multinivel" (IE1718.0606) and "QUIMETUBE: actualizando las prácticas de Química mediante Aula Invertida y TICs" (IE1718.0601), which are part of the actions implemented by the Universidad Politécnica de Madrid to support the innovative teaching.

The authors also want to acknowledge the students Polina Kushnirenko and Domenica Rosenschein, for their technical assistance in this study.

\section{REFERENCES}

[1] L. Fernández-Gutiérrez del Alamo, D. Bolonio, M. Izquierdo, F. Barrio-Parra, L.F. Mazadiego, A. Fidalgo Blanco, "The Use of Heterogeneity to Improve the Learning Process of Large Groups of Students," Sixth Edition Technological Ecosystems for Enhancing Multiculturality (TEEM18) Proceedings. 2018. ACM Press. 794-798. DOI: 10.1145/3284179.3284297 
[2] S. Abeh, D. Molenaar, K.A. Feensra, H.C.J. Hoefsloot, B. Teusink, J. Heringa, J. "Bioinformativs and Systems Biology: bridging the gap between heterogeneous student backgrounds". Briefings in bioinformatics. Vol 14. 5, 589-598. doi:10.1093/bib/bbt023. 2013.

[3] F. Pranger, "Potentials and limitations of peer-learning in small groups with respect to the homogenisation of heterogeneous learning collectives". International Journal for Lesson and Learning Studies, Vol. 5 Iss 1 pp. 19 - 35. http://dx.doi.org/10.1108/IJLLS-09-2015-0031. 2016

[4] M. Zamani. "Cooperative learning: Homogeneous and heterogeneous grouping of Iranian EFL learners in a writing context". Cogent Education, 3: 1149959. http://dx.doi.org/10.1080/2331186X.2016.1149959. 2016

[5] A. Fidalgo."Formación personalizada: Nivelación de conocimiento". https://innovacioneducativa.wordpress.com/2017/03/25/formacion-personalizada-nivelacion-deconocimiento/. 2017

[6] M.M. Crocitto, L. Walsh, A. Rphy, A. Keefe. "Diversity as a natural occurrence: an enrichment strategy of peer learning". Interactive Learning Environments. DOI: 10.1080/10494820.2017.1283330. 2017

[7] T. TangWee, D.T. Kim Chwee, Y. YawKai, T. YongChua and Y. LeckWee. Howflipteachingsupportsundergraduatechemistrylaboratorylearning.Chem. Educ. Res. Pract. 15, 550-567. 2014

[8] L. Fernández Gutiérrez del Álamo, A. Fidalgo-Blanco, J.A. Ramírez Masferrer. Uso de la Adaptabilidad en el aprendizaje de la Expresión Gráfica. Servicio de Publicaciones Universidad de Zaragoza. http://repositorio.grial.eu/handle/grial/1012. 2017 